\title{
Sequence-complementarity dependent co-assembly of phosphodiester-linked aromatic donor-acceptor trimers
}

\author{
Nadeema Appukutti, Alex H. de Vries, ${ }^{b}$ Prashant G. Gudeangadi, ${ }^{a}$ Bini R. Claringbold, ${ }^{a}$ Michelle \\ D. Garrett, ${ }^{c}$ Michael R. Reithofer ${ }^{d}$ and Christopher J. Serpell*a
}

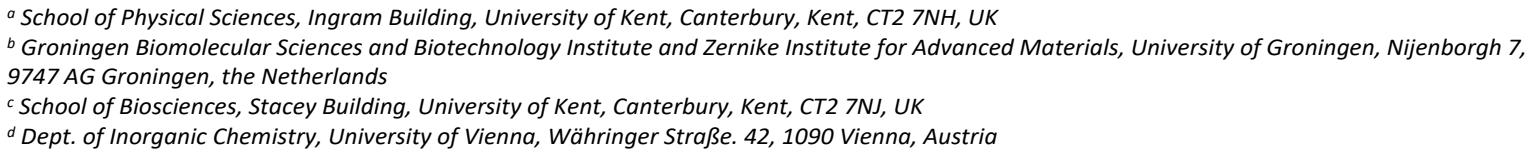

Abstract. Development of the interplay between monomer sequence and supramolecular chemistry is critical if chemistry is to recapitulate the properties of proteins and nucleic acids in the synthetic world. We have created sequenced trimers of aromatic donor/acceptor units which participate in charge-transfer interactions, linked by phosphodiesters. Each sequence displays its own characteristic self-assembly, and moreover complementary sequences interact with each other to produce new nanostructures and emergent thermochromism. This finding paves the way towards new functional nanomaterials which make bio-analogous use of sequence to tune structure.

\section{Introduction}

Sequence is the defining factor in the folding and self-assembly of biomolecules to create the functional superstructures which underpin biochemistry. On this basis, the integration of sequence into supramolecular and polymer chemistry has the potential to open up new possibilities for recapitulation of pseudobiological function in synthetic chemistry. ${ }^{[1]}$

Aromatic donor-acceptor (DA) interactions, ${ }^{[2]}$ involving electron-rich donors, such as dialkoxynaphthalene (DAN) and electron-poor acceptors, such as naphthalene diimide (NDI) have been used widely in synthetic supramolecular chemistry, ${ }^{[3-}$ 7] but are rare in biology which prefers hydrophobic collapse and hydrogen bonding. Aromatic DA interactions have been used to create chains which are capable of directed folding and selfassembly. For example, amide-linked trimers of DAN and NDI fold according to sequence, ${ }^{[8]}$ while longer alternating systems fold and self-assemble, ${ }^{[9]}$ and DA interactions have also been used to read oligomer sequences. ${ }^{[10]}$ Homooligomeric DA chains can be interweaved to create complexes ${ }^{[11-13]}$ but efforts on integrating sequence in oligomers has focussed on hydrogen bonding, ${ }^{[14]}$ metal coordination, ${ }^{[15]}$ and reversible covalent interactions. ${ }^{[16]}$ Integration of sequence into self-assembly of DA oligomers therefore raises the possibility of creating biopolymer-like superstructures using chemical motifs rare in biology. Such selfassembled materials could find applications in nanoelectronics ${ }^{[17]}$ and light harvesting, ${ }^{[18]}$ where precise electronic communication between aromatic units on the nanoscale is key.

The phosphodiester backbone of nucleic acids provides an attractive platform for the creation of sequence-defined molecules because of the outstanding efficiency of the automated phosphoramidite synthesis which permits creation of polymers of $>100$ monomer units long. ${ }^{[19,20]}$ DA interactions have been integrated into DNA itself, ${ }^{[21]}$ and the phosphodiester backbone has been used in development of various $\pi$-stacked folded and self-assembled systems, generating significant complexity from oligomers as short as trimers. ${ }^{[22-24]}$ Since aromatic donor and acceptor units are complementary in a supramolecular sense, they reflect the hydrogen-bonded complementarity of nucleobases, and their sequence in a chain could therefore be used to control self-assembly. We now report that the self-assembly of phosphodiester-linked trimers of DAN and NDI is sequence-determined, and moreover can be modified through complementarity of monomer sequences. This provides an opportunity to develop polymers in which information-rich sequences control their resultant nanostructures, creating synthetic, bio-orthogonal analogues to DNA hybridisation, which could be used in nanoelectronics or light harvesting.

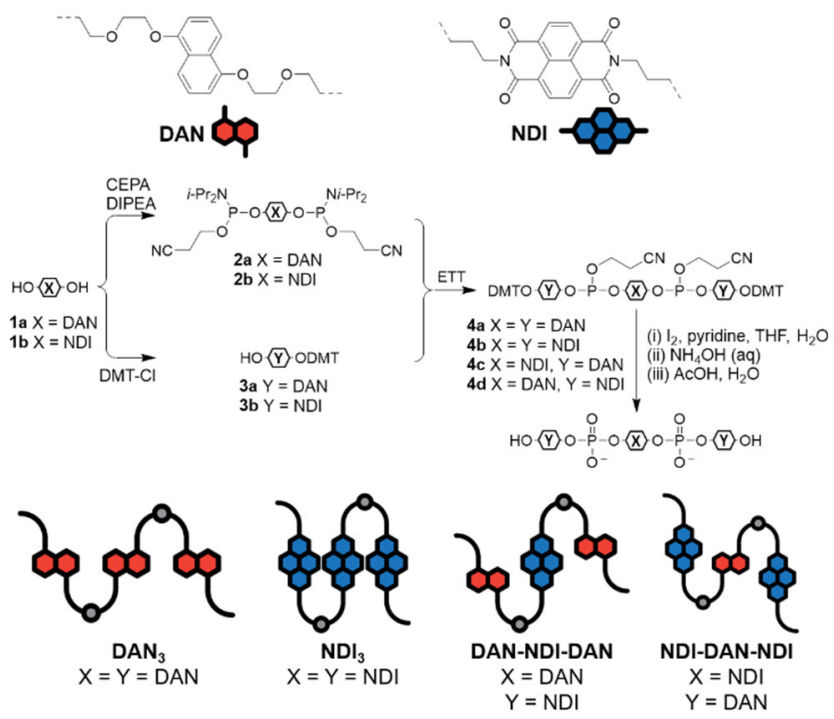

Scheme 1. Synthesis of phosphodiester trimers $\mathrm{DAN}_{3}, \mathrm{NDI}_{3}, \mathrm{DAN}$ NDI-DAN, and NDI-DAN-NDI.

\section{Results and Discussion}

DAN and NDI-containing phosphodiester-linked trimers were constructed in a symmetrical building-outwards strategy, in four sequences - DAN $_{3}, \mathbf{N D I}_{3}$, DAN-NDI-DAN, and NDI-DAN-NDI (Scheme 1). Monomers were constructed by attaching short spacers at either end of the aromatic units, terminated in 
hydroxyl units which could be used later to form phosphodiester linkages. The DAN diol monomer 1a was synthesised by reacting 1,5-dihydroxynaphthalene with chloroethyl ethanol (52\% yield) and the NDI diol $\mathbf{1 b}$ was synthesised reacting 1,4,5,8naphthalenetetracarboxylic dianhydride and 3-aminopropanol in water, expediting a reported synthesis ${ }^{[25]}$ by heating in a microwave giving the product in $70 \%$ yield and high purity. The central monomers were converted into doubly activated phosphoramidites $\mathbf{2 a}$ and $\mathbf{2 b}$ using cyanoethyl diisopropylchlorophosphoramidite (CEPA) in basic conditions, while different portions of $\mathbf{1 a}$ and $\mathbf{1} \mathbf{b}$ were dimethoxytrityl (DMT)protected on just one hydroxyl to give the terminal monomers $\mathbf{3 a}$ and $\mathbf{3 b}$. $\mathbf{2 a}$ and $\mathbf{2 b}$ were then activated immediately after synthesis with ethylthiotetrazole (ETT) and reacted separately with each of $\mathbf{3 a}$ and $\mathbf{3} \mathbf{b}$ to obtain DMT and cyanoethyl-protect phosphite triester-linked trimers in the desired sequences (4a-d). These were converted into phosphodiesters by iodine oxidation, giving a stable, organic soluble compounds which were purified by column chromatography. The cyanoethyl groups were removed under basic conditions, and final removal of the terminal DMT groups was achieved in $80 \%$ acetic acid, yielding DAN $_{3}, \mathbf{N D I}_{3}$, DAN-NDI-DAN, and NDI-DAN-NDI in 37-53\% isolated yields. All of the trimers were found to be insoluble in organic solvents, and their self-assembly in water precluded molecular analysis by NMR. Nonetheless, we were able to confirm identity by mass spectrometry (Fig. S1-S4, ESI). Molecular dynamics (MD) simulations using a modification of the united-atom Gromos force field, ${ }^{[26]}$ which is well parameterised for aromatic DA interactions, ${ }^{[27]}$ indicated that the preferred conformations of all three trimers were folded, with the mixed trimers displaying an overall S-shape which permitted the expected DA interactions between the DAN donors and NDI acceptors (Fig. 1, Fig. S23, ESI). (a)

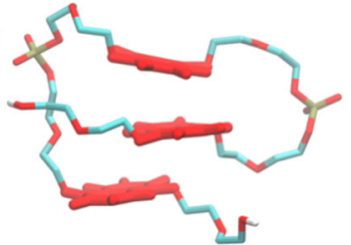

(c)

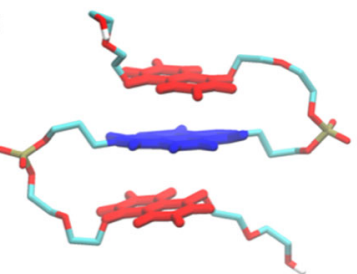

(b)

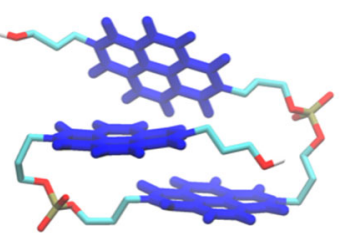

(d)

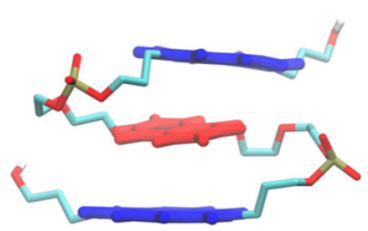

Figure 1. MD snapshots of dominant conformations of (a) $\mathbf{D A N}_{3}$ (representing $26 \%$ of structures), (b) $\mathrm{NDI}_{3}(20 \%)$, (c) DAN-NDIDAN (73\%), and (d) NDI-DAN-NDI (76\%) in water as individual molecules. DAN and NDI cores are highlighted by colouring them red and blue, respectively. Water and counter ions are not shown.

UV-visible spectroscopy is a valuable tool in characterisation of the supramolecular chemistry of DAN/NDI complexes because the DA interaction can give rise to a charge transfer (CT) band with a peak at around $525 \mathrm{~nm}$, giving the solutions a red-purple colour. The UV spectra of the homotrimers $\mathbf{D A N}_{\mathbf{3}}$ and $\mathbf{N D I}_{3}$ were recorded in water, and in tris-borate-EDTA (TBE), and Tris- acetate-magnesium (TAMg) buffers (Fig. 2a, b and S5-S6, ESI). The magnesium-containing buffer was employed since the presence of the divalent cation can have dramatic effects upon the selfassembly of oligophosphates. ${ }^{[20,28]}$ The yellow solutions gave absorption spectra characteristic of their respective aromatic units (250 - $350 \mathrm{~nm}$ for DAN, 370-400 nm for NDI), with the buffer systems making minimal difference. Both DAN-NDI-DAN and NDI-DAN-NDI gave purple solutions at $250 \mu \mathrm{M}$ in water, TBE, or TAMg (Fig. 2c, d and Fig. S8-S9, ESI), as would expected since the contain both donor and acceptor aromatics and could fold or selfassemble to satisfy DA interactions. The UV-visible spectra of both of the heterotrimers exhibited the $\mathrm{CT}$ band absorbances (450-700 nm) and no significant changes were observed for the different buffers or temperatures. The stable CT absorbance at 45 ${ }^{\circ} \mathrm{C}$ suggests that DA complex formation in these compounds is stable, while the consistency between buffers shows that the aromatic DA interactions and hydrophobic effect outweigh aspects of phosphate-phosphate repulsion. The CT band intensity was greater in DAN-NDI-DAN than in NDI-DAN-NDI; this absorption difference might be a result of NDI being a potent $\pi$ conjugated electron acceptor, while DAN is a relatively mediocre donor (2:1 DAN:NDI complexes being more favoured than the inverse ${ }^{[29]}$ ) When more DAN units are present in the chain, the donor ability increases to fulfil the acceptance capacity of NDI, but when there are more NDI units present, they each operate at a lower relative capacity.
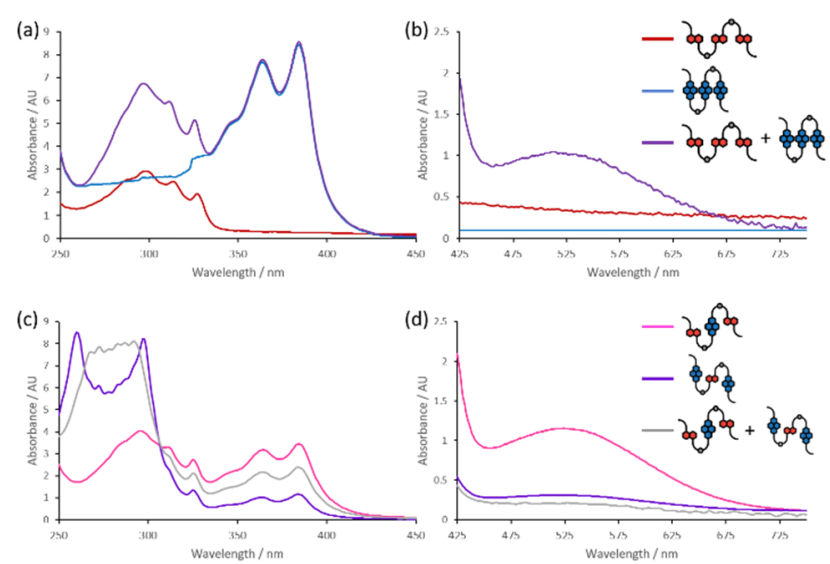

Figure 2. UV-visible absorbance spectra in water of (a-b) homotrimers and heterotrimers (c-d) highlighting typical DAN/NDI absorption bands $(a, c)$ and charge transfer bands $(b, d)$.

The self-assembly of the trimers was examined by atomic force microscopy (AFM) and transmission electron microscopy (TEM), as deposited from water (Fig. 3, and Fig. S11-S14, S17-S20, ESI), and the findings were compared with atomistic MD simulations of 48 trimer molecules in explicit water (Fig. 3, and Fig. S24-S25, ESI). Deposition of $\mathrm{DAN}_{\mathbf{3}}$ resulted in a film, with spherical protrusions of $6-20 \mathrm{~nm}$ in height (average $10.1 \mathrm{~nm}$ ) and $100-150$ $\mathrm{nm}$ in diameter. Modelling described an uneven flat layer 2-3 nm wide with the phosphate groups exposed. This is consistent with formation of the film as a bilayer, with the round protrusions being fully or partially formed vesicles developing from or collapsing into the bilayer. AFM and TEM of $\mathbf{N D I}_{3}$ showed a dense network of angular, elongated structures, averaging $7.1 \mathrm{~nm}$ in height and of various lengths between 150 and $600 \mathrm{~nm}$. In 
contrast to the smooth perimeter of the $\mathrm{DAN}_{3} \mathrm{MD}$ model aggregate, $\mathbf{N D I}_{3}$ formed finer, more angular structures, in line with the experimental data. AFM and TEM of DAN-NDI-DAN revealed small assemblies, with jagged edges, of average height $1.8 \mathrm{~nm}$ and diameter $88 \mathrm{~nm}$ - the small height is consistent with the interphosphate distance seen in the MD model of the same molecules which produced small branched sheets. AFM imaging of NDI-DAN-NDI showed smooth-edged particles and flakes (7.9 $\mathrm{nm}$ average height) of various diameters, including areas of continuous film at the same constant height, with some evidence of texture. TEM revealed this texture in more detail - the film has a sponge-like texture with distances of ca. $15 \mathrm{~nm}$ separating the higher contrast regions. MD simulations gave highly branched aggregates, which could fit with the network forming the solid part of the sponge-like aggregate.
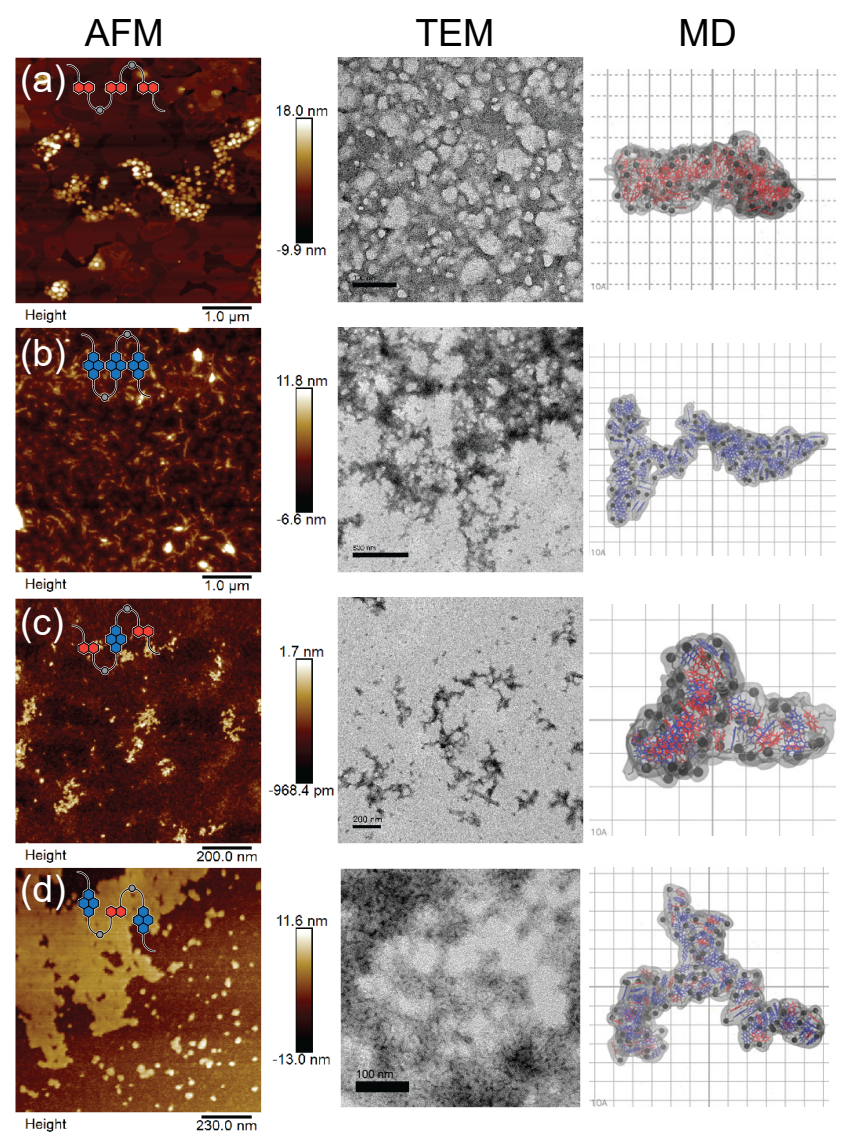

Figure 3. AFM, TEM, and MD modelling of (a) $\mathrm{DAN}_{\mathbf{3}}$ (b) $\mathbf{N D I}_{3}$ (c) DAN-NDI-DAN (d) NDI-DAN-NDI. Red units in the MD models are DAN, while blue are NDI. Dark spheres represent phosphates. The vanderWaals surface is shown as a transparent envelope. Further AFM can be found in Fig S11-S14, and TEM in Fig S17-S20, ESI

In all these cases, although the MD models exhibited some characteristic distances, overall morphologies, and short-range structural motifs, there was very little long range order, in contrast to what has been recently reported in a similar system. ${ }^{[9]}$ Our results are in line with historical observations of conformational variation in donor-acceptor aromatic foldamers, ${ }^{[29]}$ and make intuitive sense given the high degree of freedom imparted by the unconstrained 3-5 atom spacers between the aromatic units and the phosphates.
When the homotrimers $\mathbf{D A N}_{\mathbf{3}}$ and $\mathbf{N D I}_{\mathbf{3}}$ were mixed together in a 1:1 ratio in water, the solution turned from yellow to deep purple, and the CT band was seen in the UV-visible spectrum, indicating that the two systems were associating through complementary, spatially localised, donor-acceptor interactions. This CT band remained constant from room temperature up to $45{ }^{\circ} \mathrm{C}$, evidencing stability for the $\mathrm{CT}$ complex within the temperature range of the spectrometer. We can therefore be confident that the two complementary homotrimers are interacting. Conversely, when the two complementary heterotrimers DANNDI-DAN and NDI-DAN-NDI (which both display the CT band as a pure solution) were mixed together in a 1:1 ratio, the $C T$ band become less intense than either of the individual components, indicating an interaction between the trimers, but one which decreased the $\mathrm{CT}$ interaction. Upon heating up to $45^{\circ} \mathrm{C}$ it was found that the CT band decreased linearly by a further $28 \%$, and the solution lost its purple colour (Fig. S10). Thermochromism in aromatic CT complexes ${ }^{[30-32]}$ has been observed to occur via structural reorganisation ${ }^{[33]}$ and phase separation. ${ }^{[34]}$ Since phase separation would result in two CT-active compounds, it must be a degree of conformational reorganisation upon interaction of the two compounds which is occurring. It is known that conformational constraints can quench the $\mathrm{CT}$ band, despite the formation of nanostructures, ${ }^{[35]}$ and that weaker CT bands are correlated with less well defined relative geometries, ${ }^{[29]}$ and this may the case here, particularly upon heating.
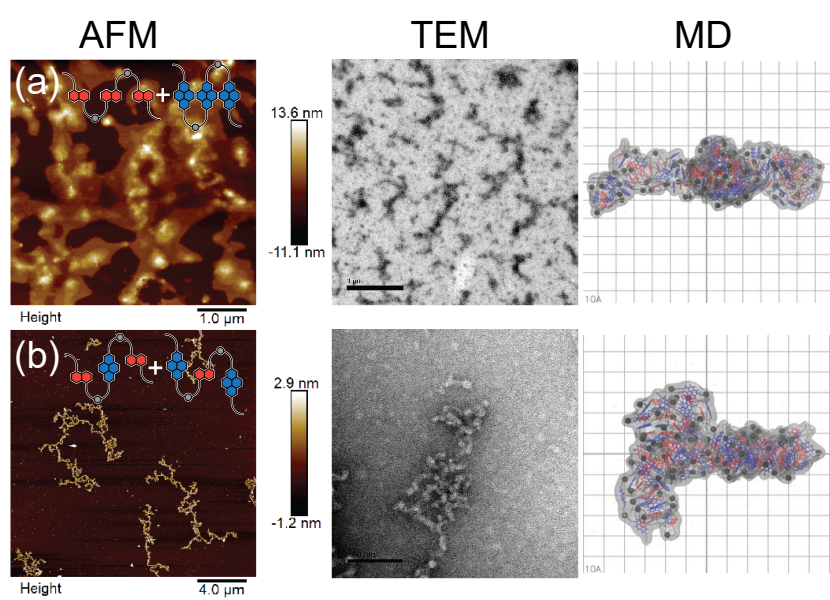

Figure 4. AFM, TEM and MD modelling of $\mathrm{DAN}_{3} / \mathrm{NDI}_{3}$ (top) and DAN-NDI-DAN/NDI-DAN-NDI (bottom) complex nanostructures. Red units in the MD models are DAN, while blue are NDI. Dark spheres represent phosphates. The vanderWaals surface is shown as a transparent envelope. Further AFM can be found in Fig S15-S16, and TEM in Fig S21-S22, ESI

Changes in self-assembly were also observed by microscopy (Fig. 4). The mixture of $\mathrm{DAN}_{3}$ and $\mathbf{N D I}_{3}$ produced a patchy film in which clear delineation of levels was seen, with steps of $2.5 \mathrm{~nm}$ measured by AFM. This spacing was also seen in MD modelling, with the two molecules joining to give a single plate-like aggregate of a very similar height, with phosphates occupying the surfaces. This is a clear change in self-assembly arising from the complementary aggregation of these two strands. The heterotrimer mixture was seen to produce a jagged, thread-like network, primarily of height 2-3 nm, although some portions were as high as $6 \mathrm{~nm}$, again in contrast to structures formed by 
either of the trimers on their own. This is consistent with the MD model which produced a structure consisting of domains with a width of 2-3 nm, but displaying branching regions. Within the MDmodelled clusters, alternating DAN/NDI stacks were observed on a small scale, but not over longer distances, showing that only local order is present, which might be easily lost upon heating, leading to reduction of the $\mathrm{CT}$ band.

\section{Conclusions}

We have synthesised sequence-defined DAN-NDI phosphodiester trimers using phosphoramidite chemistry. The sequence of aromatic donors and acceptors determines the self-assembly of each strand, through pi-stacking and charge transfer interactions. When complementary sequences are combined, new selfassembled structures, and thermochromic behaviour, emerge. Although these structures lack long-range order, short-range motifs are sufficient to influence the overall nanostructure. Since phosphoramidite chemistry enables the synthesis of long, information rich strands, this raises possibilities for new nanostructures based upon non-biological sequence recognition, resulting in systems which display the potential of DNA nanotechnology, while remaining orthogonal to biology.

\section{Acknowledgments}

P. G. Gudeangadi thanks the European Commission for a Marie Salomea Skłodowska Curie Individual Fellowship (PepDNA-4D ref 842971). C. J. Serpell, B. R. Claringbold, and M. D. Garrett thank the Rosetrees Trust for a PhD Grant (ref M743). T. K. Piskorz is acknowledged for stimulating discussion of the molecular modelling.

\section{Supporting Information}

Synthetic protocols, spectral and microscopic data, and details of molecular modelling can be found in the Supporting Information.

\section{Conflicts of interest}

None to declare.

\section{References}

[1] J.-F. Lutz, M. Ouchi, D. R. Liu, M. Sawamoto, Science 2013, 341, 1238149-1238149.

[2] C. A. Hunter, J. K. M. Sanders, J. Am. Chem. Soc. 2002, 112, 5525-5534.

[3] R. S. Lokey, B. L. Iverson, Nature 1995, 375, 303.

[4] R. S. Forgan, J. J. Gassensmith, D. B. Cordes, M. M. Boyle, K. J. Hartlieb, D. C. Friedman, A. M. Z. Slawin, J. F. Stoddart, J. Am. Chem. Soc. 2012, 134, 17007-17010.

[5] H. Y. Au-Yeung, G. D. Pantoş, J. K. M. Sanders, J. Org. Chem. 2011, 76, 1257-1268.

[6] A. Das, S. Ghosh, Angew. Chem. Int. Ed. 2014, 53, 20382054.

[7] M. E. Dehkordi, V. Luxami, G. D. Pantoş, J. Org. Chem. 2018, 83, 11654-11660.

[8] G. J. Gabriel, S. Sorey, B. L. Iverson, J. Am. Chem. Soc. 2005, 127, 2637-2640.

[9] K. P. de Carvasal, N. Aissaoui, G. Vergoten, G. Bellot, J.-J. Vasseur, M. Smietana, F. Morvan, Chem. Commun. 2021, DOI 10.1039/D1CC01064F.
[10] Z. Zhu, C. J. Cardin, Y. Gan, C. A. Murray, A. J. P. White, D. J. Williams, H. M. Colquhoun, J. Am. Chem. Soc. 2011, 133, 19442-19447.

[11] G. J. Gabriel, B. L. Iverson, J. Am. Chem. Soc. 2002, 124, 15174-15175.

[12] Q. Z. Zhou, M. X. Jia, X. Bin Shao, L. Z. Wu, X. K. Jiang, Z. T. Li, G. J. Chen, Tetrahedron 2005, 61, 7117-7124.

[13] S. De, D. Koley, S. Ramakrishnan, Macromolecules 2010, 43, 3183-3192.

[14] B. Gong, Acc. Chem. Res. 2012, 45, 2077-2087.

[15] M. Morisue, Y. Hoshino, K. Shimizu, M. Shimizu, Y. Kuroda, Chem. Sci. 2015, 6, 6199-6206.

[16] D. Núñez-Villanueva, C. A. Hunter, Acc. Chem. Res. 2021, 54, 1298-1306.

[17] A. A. Sagade, K. V. Rao, U. Mogera, S. J. George, A. Datta, G. U. Kulkarni, Adv. Mater. 2013, 25, 559-564.

[18] M. Probst, S. M. Langenegger, R. Haner, Chem. Commun. 2014, 50, 159-161.

[19] N. Appukutti, C. J. Serpell, Polym. Chem. 2018, 9, 22102226.

[20] N. Appukutti, J. R. Jones, C. J. Serpell, Chem. Commun. 2020, 56, 5307-5310.

[21] B. A. Ikkanda, S. A. Samuel, B. L. Iverson, J. Org. Chem. 2014, 79, 2029-2037.

[22] S. M. Langenegger, R. Häner, ChemBioChem 2005, 6, 2149-2152.

[23] A. D. Shaller, W. Wang, A. Li, G. Moyna, J. J. Han, G. L. Helms, A. D. Q. Li, Chem. - Eur. J. 2011, 17, 8350-8362.

[24] M. Vybornyi, Y. Vyborna, R. Häner, Chem. Soc. Rev. 2019, 48, 4347-4360.

[25] B. Baumgartner, A. Svirkova, J. Bintinger, C. Hametner, M. Marchetti-Deschmann, M. M. Unterlass, Chem Commun 2017, 53, 1229-1232.

[26] B. A. C. Horta, P. T. Merz, P. F. J. Fuchs, J. Dolenc, S. Riniker, P. H. Hünenberger, J. Chem. Theory Comput. 2016, 12, 3825-3850.

[27] P. C. T. Souza, R. Alessandri, J. Barnoud, S. Thallmair, I. Faustino, F. Grünewald, I. Patmanidis, H. Abdizadeh, B. M. H. Bruininks, T. A. Wassenaar, P. C. Kroon, J. Melcr, V. Nieto, V. Corradi, H. M. Khan, J. Domański, M. Javanainen, H. Martinez-Seara, N. Reuter, R. B. Best, I. Vattulainen, L. Monticelli, X. Periole, D. P. Tieleman, A. H. de Vries, S. J. Marrink, Nat. Methods 2021, 18, 382-388.

[28] T. G. W. Edwardson, K. M. M. Carneiro, C. J. Serpell, H. F. Sleiman, Angew. Chem. - Int. Ed. 2014, 53, 4567-4571.

[29] A. J. Zych, B. L. Iverson, J. Am. Chem. Soc. 2000, 122, 8898-8909.

[30] T. Yuan, Y. Xu, C. Zhu, Z. Jiang, H.-J. Sue, L. Fang, M. A. Olson, Chem. Mater. 2017, 29, 9937-9945.

[31] V. C. Wakchaure, L. V. Pillai, Goudappagouda, K. C. Ranjeesh, S. Chakrabarty, S. Ravindranathan, P. R. Rajamohanan, S. S. Babu, Chem. Commun. 2019, 55, 9371-9374.

[32] K. Narasimha, ACS Appl. Polym. Mater. 2020, 2, 11451159.

[33] C. D. Wight, Q. Xiao, H. R. Wagner, E. A. Hernandez, V. M. Lynch, B. L. Iverson, J. Am. Chem. Soc. 2020, 142, 1763017643.

[34] P. M. Alvey, J. J. Reczek, V. Lynch, B. L. Iverson, J. Org. Chem. 2010, 75, 7682-7690.

[35] M. A. Kobaisi, R. S. Bhosale, M. E. El-Khouly, D. D. La, S. D. Padghan, S. V. Bhosale, L. A. Jones, F. Antolasic, S. Fukuzumi, S. V. Bhosale, Sci. Rep. 2017, 7, 16501. 